\title{
On The Value Of Beta Eucain And Adrenalin As A Local Analgesic In Military Surgery
}

\author{
Commentary \\ GD Maytham
}

This paper reports on the use of local anaesthetic infiltration for analgesia in soldiers undergoing a variety of procedures in the early years of twentieth century. The solution used is described as consisting of eucain and adrenalin, a synthetic alkaloid and adrenalin, a combination of similar substances still being used to this day. Eucain had fallen out of favour by 1943, having been found to be more toxic than procaine, and less effective as a surface anaesthetic than cocaine.

The author suggested a number of benefits may be derived from the use of a local anaesthetic, some of which are still relevant at the present time. The requirement for less staff than needed in cases undergoing a general anaesthetic is certainly still valid. As far as the benefit of the use of local anaesthetic in the field being regarded as enormous, due to the surgeon being alone, one would hope that in the current setting this scenario would be rather unusual. However, the use of local anaesthesia in the field setting in the form of regional blocks for lasting analgesia is of major benefit, suggesting the role, if not the importance, has changed.

It seems that with changing times, and the oft-mentioned change in the constitution of individuals, one of the points made by the author may have become less valid. Personal experience suggests not as many modern day soldiers would readily submit to operations under local anaesthesia as perhaps would in those more hardy times. This may be due to present servicemen having a greater trust in the safety of general anaesthesia, a somewhat greater desire to avoid any experience of an uncomfortable nature, or perhaps most likely a combination of the two.

The paper describes a number of procedures performed under local anaesthetic. Of interest is how the preparation of patients has changed, with the 48 hour preparation of the surgical site by shaving and cleansing having been reduced to the moments prior to incision to decrease infective complications. Another deviation from practices in the past is the avoidance of adrenalin in circumcisions, to help prevent the unfortunate complication of ischaemic necrosis (1). Apart from these two practices, the general management of patients undergoing local anaesthetic procedures is largely unchanged, with the adjuvant use of analgesia and sedation still playing a large part in procedures undertaken using this method of anaesthesia. A further difference is seen, however, in the monitoring of the patients undergoing procedures, with this aspect not being mentioned in the article and much emphasis being placed upon this in the present day.

The use of local anaesthesia during open appendectomy is of interest. Little evidence is available on the use of local anaesthesia as the primary modality for this procedure, with only one paper describing a minimally invasive open short-stay technique, suggesting that this practice is not common (2). At present, local anaesthesia is most associated with appendectomy in the form of pre-incisional and incisional infiltration for post-operative pain relief. Various trials have investigated the efficacy of these practices in decreasing post-operative pain and length of hospital stay, with contrasting results (3-6). However, recent reports of successful laparoscopic removal of the appendix following conscious pain mapping may indicate that appendectomy under local anaesthesia is a viable option, and may become more widespread with increasing experience (7).

With the exception of removal of varices, most of the procedures listed are practiced commonly under local anaesthesia to this day. A notable inclusion is the repair of inguinal herniae, of which many are repaired under local anaesthesia in the day case setting, with this being a valuable option in patients unfit to safely undergo a general anaesthetic. The use of local anaesthesia for this reason is understandably not mentioned, with the patient base involved, although the author hints that the option of local anaesthesia would avoid the check associated with an anaesthetic related death.

At the outset, the author suggested the possibilities of the use of local anaesthesia in Army practice were very great. It, therefore, probably would not have surprised him were he to know how widespread the use of this technique in both civilian and military practice has become. 


\section{References}

1. Parfitt, K. ed. Martindale : the complete drug reference. 32 ed. London: Pharmaceutical Press, 1999.

2. Janik JS, Janik JE. Short-stay open appendectomy. Eur $\mathcal{F}$ Pediatr Surg. 2004 Feb;14(1):25-8.

3. Foulds KA, Beasley SW, Maoate K. Factors that influence length of stay after appendicectomy in children. Aust N Z F Surg. 2000 Jan;70(1):43-6.

4. Cervini P, Smith LC, Urbach DR. The effect of intraoperative bupivacaine administration on parenteral narcotic use after laparoscopic appendectomy. Surg Endosc. 2002 Nov;16(11): 1579-82.
5. Lohsiriwat V, Lert-akyamanee N, Rushatamukayanunt W. Efficacy of pre-incisional bupivacaine infiltration on postoperative pain relief after appendectomy: prospective double-blind randomized trial. World f Surg. 2004 Oct;28(10):947-50.

6. Jensen SI, Andersen M, Nielsen J, Qvist N. Incisional local anaesthesia versus placebo for pain relief after appendectomy in children - a doubleblinded controlled randomised trial. Eur $\mathcal{f}$ Pediatr Surg. 2004 Dec;14(6):410-3.

7. Almeida OD Jr, Val-Gallas JM, Rizk B. Appendectomy under local anaesthesia following conscious pain mapping with microlaparoscopy. Hum Reprod. 1998 Mar;13(3):588-90.

RAMC JOURNAL 1907 VOL VIII 361-368

\section{On The Value Of Beta Eucain And Adrenalin As A Local Analgesic In Military Surgery}

\section{by Major F J W Porter DSO Royal Army Medical Corps}

During the past twelve months the following operations have been performed at the Military

Hospital, Colchester, under the above analgesic :-

Excision of varices (chiefly of internal saphenous)

Cases

Excision of varicocele

Hammer toes (partial excision of first interphalangeal joint)

Removal of portion of dislocated semilunar cartilage $\quad 10$

Removal of large synovial fringes from knee joint

Radical cure of inguinal hernia

Radical cure of double inguinal hernia

Ingrowing toe nail (under cocaine, 1 per cent.)

Removal of glands

Evacuation of deep cervical abscess

Excision of external piles

Suture of divided tendon

Amputation of finger

Amputation of toe

Removal of sebaceous cyst

Removal of enlarged olecranon bursa

Hare lip

Excision of vermiform appendix

Excision of head of radius (for recurrent dislocation)

Circumcision

Amputation of the male breast

Excision of large ganglion

Encysted hydrocele of cord

The solution has been prepared according to Mr. Barker's formula, viz.: -

Beta eucain

Sodium chloride

Distilled water
3 grains.

12

$3^{1 / 2} \mathrm{OZ}$

Mix, boil, and, when cool, add adrenalin chloride $(1-1,000)$ "lx. 
In January, 1905, I was much struck by an operation which was performed at University College Hospital by Mr. A. E. Barker, under the above analgesic, and it occurred to me that the possibilities of this analgesic in Army practice were very great.

At first, owing to imperfect methods, one was not very successful; but as time went on one became more expert, and now it is quite exceptional to have to employ a general anaesthetic in this hospital.

The labour which is involved in nursing cases which have been operated upon under this method is, as may be readily understood, very much lessened.

In peace times the operations which are required in military hospitals are not of a very varied character, and I am strongly of opinion that the great majority can be performed quite satisfactorily under this method of local anaesthesia. I also think the soldier will more readily submit to operations of expediency if he knows they can be performed without " putting him to sleep". In small hospitals, if this method be adopted, it will be possible for the surgeon to undertake many operations single-handed. The only assistance he will require will be that of a trained orderly. In the field, or in naval practice, where the surgeon may be alone, the value of this analgesic must be enormous. For country practitioners one cannot conceive anything more valuable. It enables small operations to be done for patients who are unable or unwilling to go to the expense of an anaesthetist and a couple of trained nurses, or to expose themselves to the risk of a general anaesthetic.

A marked feature of these operations is their bloodlessness.

In using this drug the main points to be attended to are: (1) To provide oneself with a reliable syringe, which will not be out of order at the time it is required ; (2) to make the solution freshly and so ensure its sterility; (3) soda must not be brought in contact with it for fear of decomposing the adrenalin; (4) to inject sufficient. It is quite possible to inject as much as 6 or 7 ozs. with safety. For the removal of the whole of the axillary glands on one side not less than 8 ozs. were used. It is quite a mistake to use the small quantities to which one was accustomed when making use of cocain solutions; (5) to wait for at least twenty minutes before operating. The injected parts are much less oedematous, and the landmarks are much less obscured, if one waits for three-quarters of an hour ; (6) to use drainage for the first eighteen hours in all large operation wounds. Owing to the adrenalin the blood-vessels are emptied, and they remain in this condition for some considerable time. They are placed in very much the same condition as when an Esmarch bandage and tourniquet are applied to a limb. The natural consequence is that when the blood is readmitted a good deal more exudation occurs than when the same operation is done under a general anaesthetic. The tissues may be able to deal with this fluid, but on the whole I think it is safer to make use of a drain for a few hours in all the larger operation wounds. Four strands of coarse silkworm gut answer perfectly ; (7) to operate as gently and as tenderly as possible. Much tearing and dragging cannot be tolerated.

I should like to describe some of the operations which have been performed in detail.

Varicose Veins - The skin of the affected parts is carefully prepared for at least two days, by shaving and thorough cleansing with turpentine and spirit soap. A carbolic compress is then applied and the patient walks to the theatre. The skin is finally cleansed, and the man is made to put all his weight on the affected limb. With carbolic fuchsin the outline of the dilated veins is marked on the skin, and also the upper part of the internal saphenous vein. In the case of varix of the external saphenous vein, the upper part of this is marked out. The eucain and adrenalin solution is then injected into the subcutaneous tissue round the affected veins for about half an inch on each side, and for one inch above and below the limit of the section which it is proposed to remove. The limb is then wrapped in a sterilised towel and the operation is not commenced for at least twenty minutes. The internal saphenous vein is then exposed through a transverse incision about $1 \frac{1}{2}$ inches long, and tied about 3 or 4 inches below the opening. In fat subjects, where the vein is not dilated at this point, this is by no means as simple an operation as it appears, and the vein is sometimes very difficult to find. This incision is sutured at once by continuous very fine silkworm gut and a dressing applied. A series of small incisions about three-quarters of an inch in length are then made at right angles to the line of the dilated veins and about 3 inches apart, commencing below. The exposed veins are clamped by Spencer Well's forceps, and cut across. By gentle traction, aided by a Watson Cheyne dissector, the veins are separated from their connective tissue bed as high up as possible, small tributary veins are clamped, cut across, and twisted and pulled until they break off. Through the next incision higher up the vein is clamped and cut across. The lower part is freed towards the lower incision as far as possible, and once forceps have been removed, the section is twisted out. The same procedure is adopted until all the dilated veins have been dealt with. It is advisable to close the incisions as they are finished with by continuous silkworm gut sutures, so as to avoid possible infection of these wounds. Fresh dressings are then applied, and a back splint with foot piece is kept on for at least fourteen days. Spirit soap 
removes the fuchsin, so it must not be used to mark out the veins until the final cleansing has been carried out. In cases of varix of the external saphenous, this vein should be ligatured before its entrance into the popliteal vein. The amount of disturbance of the patient's skin and subcutaneous tissue which is involved by this method of operating, is very small as compared with the usual plan of making one long, or a series of long, incisions, and dissecting out the veins with their surrounding subcutaneous fat. No preliminary injection of morphia is usually required and no drainage is necessary for these cases.

Varicocele - The subcutaneous tissue over the external ring, and just below it, is freely injected. The fine needle is then passed behind the cord close to the pubic crest, and about 3 drachms injected here. After an interval of about fifteen minutes, an incision about $1 \frac{1 / 2}{2}$ inches is made vertically below the external ring, and the cord is lifted from its bed and pulled en masse out of the wound. Some more solution is now injected into the cord upwards and downwards. After a further interval of about ten minutes, the superfluous vessels are tied with fine kangaroo tendon and a section of about 2 inches removed. The ends of the upper and lower ligatures are tied together and a few points of catgut inserted so as to bring the cut ends of the vessels into apposition. The operation is completed by uniting the skin by a fine continuous silkworm gut suture. No morphia is required and drainage is unnecessary. The scrotum must be well supported, otherwise it becomes oedematous. About 1 ounce of solution is necessary for this operation.

Inguinal Hernia. - Before commencing to inject, give 6 minims of morphia hypodermically. In all large operations, and especially in nervous subjects, this will be found of very great assistance. It removes all fear and the natural expectation that some pain will be felt. Several patients have actually gone to sleep during the operation. A small patch of skin is injected just below the external ring, and after five minutes a fine trocar is pushed through this ansaesthetic spot. The long blunt needle, with eye near the end, is then pushed through this puncture, and while the fluid is being injected it is sent in three or four different directions so as to flood the whole of the subcutaneous tissue of the operation area; about 1 ounce is required for this. After an interval of about fifteen minutes, the tissues are divided down to the external oblique aponeurosis. The blunt needle is then pushed up the inguinal canal, and about 6 drachms injected. After waiting for ten or twelve minutes, the inguinal canal is laid open and the sac isolated. Owing to the action of the adrenalin, the sac is blanched and is identified very easily. Personally, I never strip up more sac than is required to push through the internal oblique after it has been transfixed and tied, but if the prolongation, which may extend into the scrotum, is removed, more solution must first be injected downwards into the cord. It is usually found that the sac can be stripped painlessly until a point is reached near the internal ring. It is advisable now to inject a little more fluid into the substance of the sac and into the internal oblique muscle. While this is acting $\mathrm{I}$ introduce the kangaroo sutures through the conjoined tendon and external oblique aponeurosis near Poupart's ligament, after MacEwen's method. The stripping of the sac and its transfixion and tying may now be completed. The stump of the sac is then pulled up through the separated muscle bundles of the internal oblique, twisted, and sutured in that position. The operation is then completed in the usual manner, and a silkworm gut drain put in at the highest part of the skin incision. This is, of course, the most dependent point when the patient is lying in bed with the thigh flexed. For an ordinary case, not less than 3 ounces should be injected, while if the subject is fat or the sac very thick and adherent, 4 or 5 ounces may be required.

Dislocated Semilunar Cartilage - In this operation, the subcutaneous tissue is injected through a fine needle, and if the synovial membrane is found to be very thick or sensitive when grasped in a pair of forceps, it is advisable to inject some more fluid into it, and to wait for five minutes before proceeding further. Synovial fringes should be further injected before cutting them away. Morphia and drainage of the subcutaneous tissue only are required.

External Piles - After a preliminary injection of morphia, inject the skin round the anus freely. The fine needle is then pushed into the pile and also into the mucous membrane at their inner side. After injecting, wait fifteen minutes. The pile is then dissected out, and the edges of skin and mucous membrane united by means of a fine curved needle and thin catgut. The operation is practically bloodless.

Hammer Toe - Amputation of the whole of the second toe (which is the one usually affected) almost invariably leads to an increase of the Hallux valgus, which is commonly present, and a painful bunion. Complete excision of the first interphalangeal joint leads to a stiff toe. I have for some time past practised a very simple operation, which has given excellent results. The affected toe is freely injected with the solution, care being taken that it travels well into the sole and up into the dorsum. After an interval of fifteen minutes, the knife is entered on the inner side of the toe, over the lateral ligament connecting the first two phalanges. It is carried in a curved direction through the skin as far as the outer side of the joint. The knife is again entered on the inner 
side, and carried with a curve in the opposite direction to meet the end of the first incision. The included piece of tissue containing the skin, with the usual corn and bursa, is removed. The extensor tendon and lateral ligaments are divided. It is not advisable to remove much of the extensor tendon, otherwise it will be found (owing to retraction) difficult to suture. The second and third phalanges are now forcibly depressed. This causes the head of the first phalanx to project into the wound. By means of bone forceps it is freely removed. It will now be found that when the toe is extended, the edges of the skin come into accurate apposition. The divided tendon is now sutured with fine silk, and some of the fibrous structures by means of the same material. The skin is united by finest silkworm gut. A straight splint is applied so as to keep the joint slightly over-extended. The result is a shortened toe, but it has perfectly movable joints. Care should be taken not to divide the nerves which run along the sides of the toe. The patient need not be kept in bed for more than a few days, after which he manages to get about on his heel. The splint is discontinued at the end of the third week, and by the end of the fourth he can wear his boots and walk comfortably. In very exceptional cases it may be necessary to divide the flexor tendon. The section of the bone is quite painless.

Removal of Vermiform Appendix - Recently, five appendices have been removed under eucain. Six minims of morphia are first given hypodermically. Then a small patch of skin just above the anterior superior spine of the ilium is injected. After an interval of five minutes a fine trocar is pushed through the skin, and through the opening the long blunt needle is introduced, and the subcutaneous tissue of the operation area freely injected. After fifteen minutes a $2^{1 / 2}$ inch curved incision is made, one finger's breadth above the anterior superior spine and parallel to Poupart's ligament. This is carried through the external oblique aponeurosis quite painlessly. The long blunt needle is then pushed into the internal oblique muscle at the centre of the wound, and about 1 ounce of the solution is injected in four directions, care being taken to pass a good deal into the structures near Poupart's ligament. The fluid is injected as the needle is being pushed onwards. After a delay of five or six minutes, it will be found possible to forcibly separate the muscle bundles of the internal oblique and transversalis, and to reach the peritoneum quite painlessly. This structure may still be sensitive, but usually it can be divided at once. If not, some more solution may be poured into the bottom of the wound and a few minutes' further delay given. The patients' sensations when the finger is introduced into the cavity are various. One man vomited. In all the cases it was possible to withdraw the caecum and identify the base of the appendix without very much discomfort being entailed. In two cases it became necessary to give about 30 drops of chloroform while the appendix was being withdrawn from the wound. The operation was then completed without further administration, and quite painlessly. In three cases no anaesthetic was required at all. In two more cases the appendix was so placed that its removal required full anaesthesia, after the abdomen had been opened under eucain. About $3^{1 / 2}$ ounces of solution are required, and drainage of the subcutaneous tissue is advisable for a few hours.

Circumcision - Of the 110 cases of circumcision done during the year, the first eighty were done under 1 per cent cocaine; the remainder have been done under eucain and adrenalin. No vessels require tying, and the operation is practically bloodless. If the prepuce can be fully retracted, and the solution injected into the mucous membrane near the corona, as well as into the subcutaneous tissue at the line of incision, the operation is absolutely painless. If retraction cannot be accomplished, a good deal of manoeuvring is required to flood the usually thickened mucous membrane with the solution. To accomplish this, I push the needle obliquely through the skin at the line of incision (injecting all the time), until fluid escapes from the preputial orifice. I then (while still injecting) withdraw the needle until the flow ceases, and as soon as this is noticed, the injection is continued into the mucous membrane, in which the point of the needle is now known to be embedded. This is done at two other points, and after an interval of fifteen minutes the operation can be painlessly completed. I unite the edges of the divided skin and mucous membrane by a continuous suture of fine catgut, and apply a firm gauze bandage wrung out of 1 in 1,000 perchloride. The patient grasps the penis over the cut surface for half an hour, and then lets go.

I have not found Barker's eucain syringe a satisfactory one. The rubber on the piston is not thick enough, and fluid is apt to escape above it when injecting under much pressure. The glass barrel is apt to get broken in sterilising by boiling. The aseptic needles which are supplied (size 17) are too fine, and necessitate a great deal of force to inject the solution through them into dense tissues. Size 12 are much more satisfactory, and are only about the same thickness as an ordinary hypodermic needle. Messrs. Allan and Hanbury have made me an all-metal syringe which is perfectly satisfactory. Barker's carriers are made to screw into the nozzle, so as to avoid leakage at this point.

I do not pretend that the whole of these operations have been absolutely painlessly performed. In doing the same operation in precisely the same manner on different 
subjects, one cannot help noticing that some will complain a little of the prick of even a fine needle, whereas others make no objection whatever. What I do contend is, that the amount of pain which is inflicted is very slight, and is certainly not more than a soldier should be expected to put up with. An ideal case is one in which the patient actually asks for his operation to be done under eucain. Soldiers are fond of talking about their operations, and in this way the news soon spreads through a garrison that cases can be successfully operated on in this manner. Moreover, for those who are keen on operative surgery, it is always well to bear in mind the check which would follow upon a death under a general anaesthetic in a military hospital. I am quite satisfied that many men have submitted to operative measures in this hospital lately, who would have refused to be done under general anaesthesia. 